\title{
Multiple scattering in optical coherence tomography signal: Monte Carlo modeling and experimental study
}

Gang Yao, Lihong V. Wang

Gang Yao, Lihong V. Wang, "Multiple scattering in optical coherence tomography signal: Monte Carlo modeling and experimental study," Proc. SPIE 3598, Coherence Domain Optical Methods in Biomedical Science and Clinical Applications III, (30 April 1999); doi: 10.1117/12.347489 CA, United States 


\title{
Multiple Scattering in Optical Coherence Tomography Signal: Monte Carlo Modeling and Experimental Study
}

\author{
Gang Yao, Lihong Wang* \\ Optical Imaging Laboratory, Biomedical Engineering Program, Texas A\&M University \\ 233 Zachry Engineering Center, College Station, TX 77843-3120
}

\begin{abstract}
The angle biased Monte Carlo technique is applied to simulate the OCT signal from homogeneous turbid medium. The OCT signal is divided into two categories: one is from a specific imaging target layer in the turbid medium (Class I photon); the other is from the other background medium (Class II photon). The Class II signal has wider spatial and angular distribution than the Class I signal. And it experiences more scattering events. The multiple scattered photons will decrease the contrast of the OCT image and their contributions become dominant at larger depths. The average number of scattering events increases with the probing depth for both Class I and Class II lights. Experimental study is conducted by measuring the depth-resolved degree of polarization (DOP) of the back-scattered signal from the turbid media. The DOP is derived from the Stokes vector measurements. The incident light is linear polarized and could be depolarize by the multiple scattering. The DOP decreases to 0.5 when Class I signal is equal to the Class II signal. Experiments in the Intralipid solution with different scattering coefficient show the imaging depth is limited to $3 \sim 4$ optical depths.
\end{abstract}

Keywords: Optical coherence tomography, Monte Carlo, multiple scattering, depolarization, stokes vector

\section{INTRODUCTORY}

Because of its high spatial resolution and high dynamic range, optical coherence tomography (OCT) ${ }^{1}$ has drawn much attention recently. Unfortunately, its applications in highly scattering biological tissue are limited by its penetration depth unlike its successful applications in transparent tissues such as the ocular organs. ${ }^{2,3}$ It is believed that multiple scattering that becomes dominant at large depths is the fundamental limitation for OCT to achieve a large probing depth in turbid media. ${ }^{4}$

In order to understand the governing physical process and better interpret the OCT signal in highly scattering media, a couple of theoretical models have been developed. Pan et al ${ }^{5}$ established the relationship between the path-length resolved reflectance signal with the OCT signal using linear system theory. They used Monte Carlo technique to simulate the pathlength resolved reflectance but did not separate the effects of the singly scattered light and the multiply scattered light. Recently, Schmitt et $\mathrm{al}^{6}$ described an OCT model based on the Huygens-Fresnel diffraction optics. They split the OCT signal as the summation of singly back-scattered light (coherent) and multiply scattered light (partially coherent). The effect of multiple scattering on the formation of speckle patterns and the degradation of image contrast were demonstrated.

In reality, light scattering in turbid media is a complex process, and it is only an approximation to assume that the OCT signal is from single back-scattering alone. A photon still contributes to the OCT signal after a limited number of scattering events. A more realistic model is needed to study the OCT signal from the turbid media.

In this paper, we simulate the light scattering process in homogeneous turbid media by Monte Carlo method. ${ }^{7}$ The OCT signal is divided into two classes: one is the light coming from the target layer in the medium; and the other is the light coming from the background other than the target layer. Angle biasing technique ${ }^{8}$ is applied to speed up the simulation and reduce the statistical variance. A polarization sensitive OCT is used to measure the depth resolved degree of polarization (DOP) of back-scattering signal from turbid media. The DOP is derived from the Stokes vector of the back-scattered signal. Because the multiple scattering will depolarize the incident light, the change of polarization degree is a sensitive indication of the dominance of the multiple scattering events.

\footnotetext{
* Correspondence: Email: lwang@tamu.edu, Tel: (409)847-9040, Fax: (409)845-4450
} 


\section{THEORETICAL STUDY}

\subsection{OCT model}

An OCT system is a Michelson interferometer illuminated by a low-coherence light source. The light from the reference arm and the sampling arm can interfere at the detector only when the difference of their optical path-lengths is within the coherence length of the light source. The interference signal is used to construct a tomographic image of the sample. Therefore, the spatial resolution of OCT is determined by the coherence length of the light source.

In the simulation, it is assumed that the probing fiber is in direct contact with the turbid medium. For simplicity, the fiber is assumed to emit a pencil beam. The radius of the probing fiber is $10 \mu \mathrm{m}$, and the receiving angle is $5^{\circ}$. The light back-scattered from the sample is divided into two different classes: Class $I$ and Class II (Fig. 1).

The Class I light is the light scattered from a specific layer whose central depth corresponds to the path-length of the reference arm and whose thickness is determined by

$$
2 n \Delta z=l_{c},
$$

where $n$ is the refractive index of the medium, $l_{c}$ is the coherence length of the light source in vacuum, and $\Delta z$ is the thickness of the layer. Because the photons scattered from this layer contain information about the local optical properties, the Class $I$ light is considered the useful signal that provides direct imaging

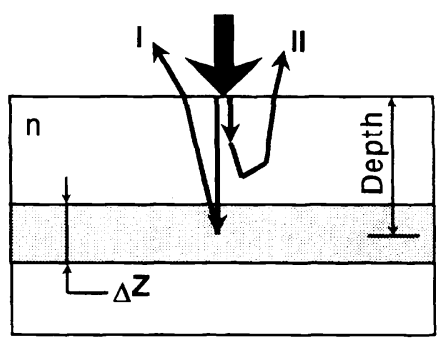

Fig.1. Composition of the OCT signal, where I represents the light from the specified target layer (Class $I$ ), and II represents the light from the rest of the medium (Class II). information. The Class II light is the light scattered from the rest of the medium whose optical path-length is within the range of $\left[p-l_{c} / 2, p+l_{c} / 2\right]$, where $p$ is the path-length of the reference arm. Because the path-length difference between this part of the light and the reference light is within the coherence length, the Class II light affects the OCT signal. The Class II light may be from anywhere above the specific layer in the turbid medium, i.e., it does not contain information about the specific probing depth in the medium. Therefore, this part of the light is responsible for the degradation of the contrast of OCT images and may overwhelm the Class I signal at large probing depths.

The OCT signal can be written as: ${ }^{5}$

$$
I_{d}(\tau)=I_{s}+I_{r}+2\left(I_{s} I_{r}\right)^{1 / 2} \operatorname{Re}\left[V_{m c}(\tau)\right],
$$

where $\tau$ is the time delay between the reference arm and the sampling arm; $I_{s}$ and $I_{r}$ are the ensemble averaged light intensities from the reference arm and the sampling arm, respectively; and $V_{m c}$ is the mutual coherence function of the light from the two arms and is assumed to be rectangular for simplicity. Equation (2) indicates that the OCT signal is proportional to the square root of the diffuse reflectance $I_{s}$. In our model, $I_{s}$ is the summation of the Class $I$ light $\left(I_{1}\right)$ and the Class $I I$ light $\left(I_{2}\right)$ :

$$
I_{s}=I_{1}+I_{2} \text {. }
$$

The light whose path-length difference with the reference path-length is beyond the coherence length is simply discarded because it does not contribute to the OCT signal.

\subsection{Angle biased Monte Carlo simulation}

Monte Carlo simulation has been proved to be an accurate method to study photon-tissue interaction. Because biological tissues usually have very large anisotropy factors, light undergoes highly forward scattering and has a small chance to be back-scattered. However, OCT modeling requires very high spatial resolution (of the order of the coherence length). Therefore, it would be a very time consuming task to use the conventional Monte Carlo algorithm. In order to accelerate the computation, we applied a variance reduction technique called "angle biased" sampling. ${ }^{8}$ The basic idea is to use an artificial scattering phase function to replace the true phase function when sampling the scattering angle and then update the photon weight according to: 


$$
w^{*}=\frac{f(\theta, \varphi)}{f^{*}(\theta, \varphi)} w,
$$

where $f(\theta, \varphi)$ is the true phase function for the photon scattering, $f^{*}(\theta, \varphi)$ is the artificial phase function used in the sampling, $w$ is the photon weight corresponding to the phase function $f(\theta, \varphi)$, and $w^{*}$ is the photon weight corresponding to the phase function $f^{*}(\theta, \varphi)$.

To implement angle biasing in our modeling, the Henyey-Greenstein phase function is chosen to sample the photon scattering angle:

$$
p(\cos \theta)=\frac{1-g^{2}}{2\left(1+g^{2}-2 g \cos \theta\right)^{3 / 2}},
$$

where $g$ is the anisotropy factor, and $\cos \theta$ is the photon deflection angle of a single scattering event. $p(-\cos \theta)$ is applied as the artificial phase function. In other words, after $\cos \theta$ is sampled with $p(\cos \theta),(-\cos \theta)$ is actually used to calculate the traveling direction of the photon packet. Meanwhile, the photon weight is adjusted by:

$$
w^{*}=\left(\frac{1+g^{2}+2 g \cos \theta}{1+g^{2}-2 g \cos \theta}\right)^{3 / 2} w .
$$

The same technique was used in the simulation of confocal microscopy. ${ }^{9}$ Our simulation results show that this method has greatly improved the statistical properties of the calculated diffuse reflectance and significantly reduced the computation time.

\subsection{Simulation process}

The specular reflection from the fiber-tissue interface is neglected in our simulation. The basic Monte Carlo simulation method is described in detail in ref. 7. In the modeling process, a layer in the medium is specified with a central depth corresponding to a predefined optical path-length in the reference arm (Fig. 1). Photons are launched and traced by the standard Monte Carlo method. Whenever a photon hits this layer, it is labeled, and its scattering angle is sampled by the angle biased sampling technique such that the back-scattering process is preferentially traced. Otherwise, the photon scattering is sampled by the normal Henyey-Greenstein phase function. The optical path-length of each photon packet is also recorded. The photon is discarded whenever the difference between its path-length and the reference path-length is beyond the source coherence length. The labeled photons reaching the detector are scored into the Class I signal and the unlabeled photons are scored into the Class II signal. The signal intensity is calculated as the square root of the diffuse reflectance, and the decay constant of the signal is calculated as the derivative of the signal intensity with respect to the probing depth. When the photons exit the surface of the turbid medium, the horizontal distance from the point of light incidence and the exit angle with respect to the normal to the surface of the medium are recorded for calculation of the spatial or angular distribution of the signal.

\section{SIMULATION RESULTS}

\subsection{Verification of angle biased Monte Carlo}

To verify the correctness of the angle biased Monte Carlo technique in this simulation, we compare the spatial and angular distributions of the Class I signal for small probing depths with those of the conventional Monte Carlo simulation. The comparison for a large probing depth is difficult because the statistical error in the conventional Monte Carlo method is too large. Figure 2 shows the results for the depth of $100 \mu \mathrm{m}$. Both simulations use $10^{8}$ incident photons. Obviously, the angle biased sampling technique yields much smoother results than the conventional approach. The calculated total reflectances by the two methods are in agreement with each other. For example, for the depth of $100 \mu \mathrm{m}$, they are $1.425 \times 10^{-5} \pm 0.037 \times 10^{-5}$ and $1.417 \times 10^{-5} \pm 0.010 \times 10^{-5}$, respectively. 

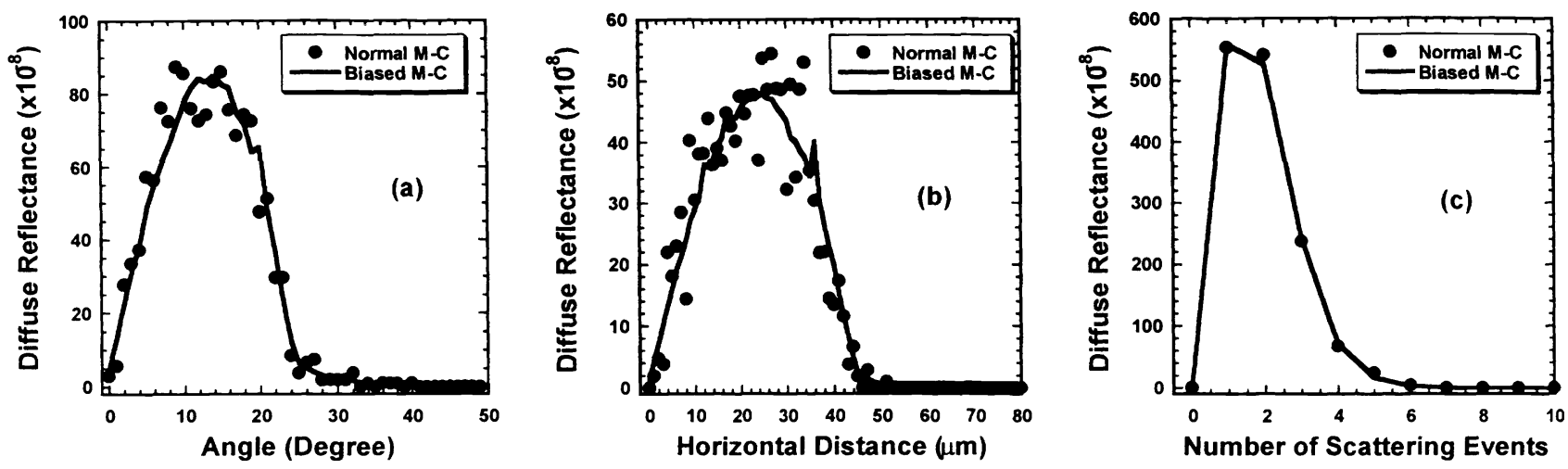

Fig. 2. Comparison of the conventional and angle-biased Monte Carlo (M-C) simulation in (a) the angular distribution, (b) the spatial distribution, and (c) the number of scattering events distribution, where $l_{c}=15 \mu \mathrm{m}, n=1.5, \mu_{\mathrm{a}}=1.5 \mathrm{~cm}^{-1}, \mu_{\mathrm{s}}=60 \mathrm{~cm}^{-1}$ and $\mathrm{g}=0.9$.

\subsection{OCT signal profiles for different probing depths}

The coherence length of the light source in vacuum is assumed to be $l_{c}=15 \mu \mathrm{m}$. The optical properties of the turbid medium are assumed as follows: the refractive index $n=1.5$, the absorption coefficient $\mu_{\mathrm{a}}=1.5 \mathrm{~cm}^{-1}$, the scattering coefficient $\mu_{\mathrm{s}}=60 \mathrm{~cm}^{-1}$, the total attenuation coefficient $\mu_{\mathrm{t}}=\mu_{a}+\mu_{\mathrm{s}}=61.5 \mathrm{~cm}^{-1}$, and the anisotropy factor $\mathrm{g}=0.9$. The Class I photon and Class II photon have very different spatial and angular distributions. As shown in Fig. 3, the Class II signal has a much broader spatial distribution than the Class I signal. Although the spatial distributions of both signals become broadened as the probing depth increases, the Class II signal is broadened much faster than the Class I signal. Usually, an OCT system has a detection area much smaller than the spatial distribution of the Class II signal, which is very helpful to reject most of the Class II signal.

If the detection area on the sample surface has a radius $r_{d}$ of $10 \mu \mathrm{m}$, the normalized angular distributions of the Class I and Class II signals with angular resolution of $5^{\circ}$ are shown in Fig. 4. Clearly, the Class II signal has a much wider angular distribution than the Class I signal. Although the absolute intensity decreases as the probing depth increases, the distribution profiles are almost the same. With an optimal detection angle, the detector should accept the Class I light as much as possible and reject the Class II as much light as possible. There is a trade off because when the detection angle is narrowed to reject the Class II photon, the received Class I signal is reduced, and vice versa.
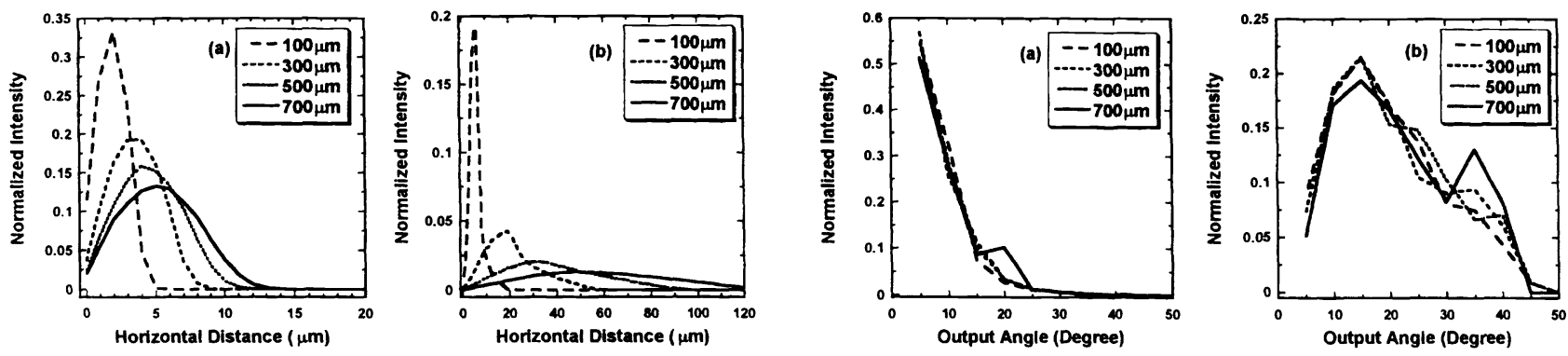

Fig.3. Normalized spatial distributions of (a) the Class $I$ and (b) the Class $I I$ signal for various probing depths, where $l_{c}=15 \mu \mathrm{m}$, $n=1.5, \mu_{\mathrm{a}}=1.5 \mathrm{~cm}^{-1}, \mu_{\mathrm{s}}=60 \mathrm{~cm}^{-1}$ and $\mathrm{g}=0.9$.

Fig.4. Normalized angular distributions of (a) the Class $I$ and (b) the Class II signal for various probing depths, where $l_{c}=15 \mu \mathrm{m}$, $n=1.5, \mu_{\mathrm{a}}=1.5 \mathrm{~cm}^{-1}, \mu_{\mathrm{s}}=60 \mathrm{~cm}^{-1}, g=0.9$ and $r_{d}=10 \mu \mathrm{m}$.

If the detection area is limited to $r_{d}=10 \mu \mathrm{m}$, and the detection angle is limited to $\theta_{d}=5^{\circ}$, then the Class II signal intensity exceeds the Class I signal intensity at the depth of approximately $500 \mu \mathrm{m}$ (Fig. 5). This critical depth, at which the Class II light intensity is equal to the Class $I$ light intensity, should actually be larger because the numbers of scattering events related with the Class II and the Class I signals are different. The Class II photons undergo more scattering events than the Class I photons. The average number of scattering events of the Class II signal increases faster with the probing depth than 
that of the Class I signal (Fig. 6). Because multiple scattering depolarizes the light, the actually measured OCT signal is less than the calculated signal intensity. In an idealized scenario where we assume that the polarization of the Class II light is completely randomized for large probing depths while the polarization of the Class I light is maintained, the critical depth becomes approximately $700 \mu \mathrm{m}$ (Fig. 5). This crossover point is important because at this point the $100 \%$ image contrast decreases to $50 \%$. When the light in both the classes is partially polarized, the critical depth is between 500 and $700 \mu \mathrm{m}$.

Figure 6 indicates that the Class $I$ light also contains multiply scattered photons. The average number of scattering events in the Class $I$ signal returned from a depth of $200 \mu \mathrm{m}$ is 2 and increases linearly with the probing depth. Owing to the requirement of matching the optical path-lengths, these multiple scattering events in the Class $I$ light must be small angle scattering. Otherwise, their optical path-length difference with the reference arm would exceed the coherence length and would not contribute to the OCT signal. Therefore, these multiply scattered Class I photons will still largely preserve their polarization properties and hence can not be rejected by the detection system. Theoretically, only the singly back-scattered photons can directly furnish the exact local optical information because they do not experience interactions elsewhere. Conversely, the multiply scattered photons do not furnish accurate local optical information because they experience interactions at multiple sites.

Figure 7 shows the decay of the Class I signal with different numbers of scattering events. The singly back-scattered light is the strongest for small probing depths but is soon exceeded by multiply scattered light as the probing depth increases. It can be seen that all the curves decay almost exponentially. The decay constant (double pass) of the singly back-scattered light is much greater than the total attenuation coefficient $\mu_{\mathrm{t}}$ In this example, the decay constant is $\sim 90 \mathrm{~cm}^{-1}$, while $\mu_{\mathrm{t}}=61.5 \mathrm{~cm}^{-1}$. This phenomenon can be easily understood by considering the geometric scheme of the OCT system in this simulation.

In general, the detected signal intensity depends on three factors: (1) the number of photons hitting the target layer; (2) how many of these photons are back-scattered; and (3) among the back-scattered photons, how many reach the detection area. For the OCT scheme used in this simulation, we can assume that the singly back-scattered photons from different depths have the same angular distribution because they have the same scattering probability distribution. The singly back-scattered light from a large depth has a large output size on the surface and hence is captured less by the detector. This effect leads to a decay constant of the singly back-scattered light greater than $\mu_{\mathrm{t}}$. For the multiply scattered component of the Class I signal, the collective effect of these factors leads to a decay constant that is slightly different from $\mu_{\mathrm{t}}$.

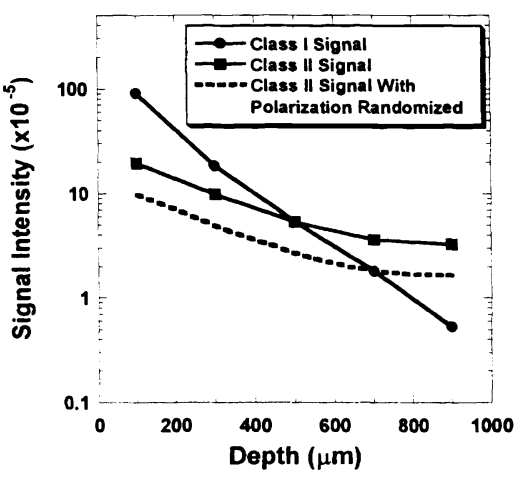

Fig.5. Classes I and Classes II signals versus the probing depth, where $l_{c}=15$ $\mu \mathrm{m}, n=1.5, \mu_{\mathrm{a}}=1.5 \mathrm{~cm}^{-1}, \mu_{\mathrm{s}}=60 \mathrm{~cm}^{-1}$, $g=0.9, r_{d}=10 \mu \mathrm{m}$ and $\theta_{\mathrm{d}}=5^{\circ}$.

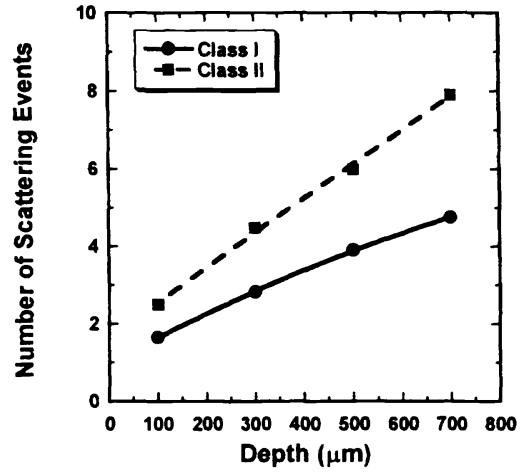

Fig.6. Weighted average numbers of scattering events of the Class I and Class II signals versus the probing depth, where $l_{c}=15 \mu \mathrm{m}, n=1.5, \mu_{\mathrm{a}}=1.5 \mathrm{~cm}^{-1}, \mu_{\mathrm{s}}=60$ $\mathrm{cm}^{-1}, g=0.9, r_{d}=10 \mu \mathrm{m}$ and $\theta_{\mathrm{d}}=5^{\circ}$.

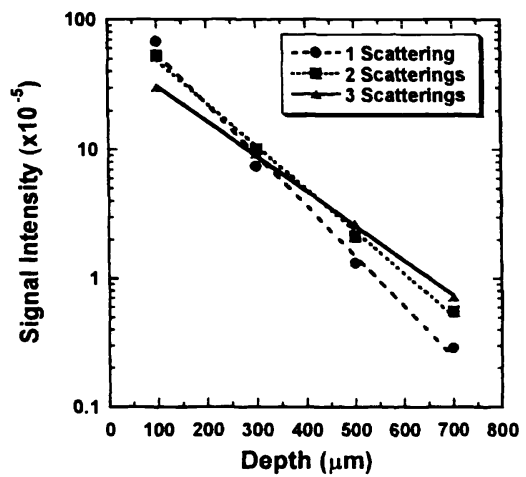

Fig.7. Decay of the Class I signal intensity with different numbers of scattering events versus the probing depth, where $l_{c}=15$ $\mu \mathrm{m}, n=1.5, \mu_{\mathrm{a}}=1.5 \mathrm{~cm}^{-1}, \mu_{\mathrm{s}}=60 \mathrm{~cm}^{-1}$, $g=0.9, r_{d}=10 \mu \mathrm{m}$ and $\theta_{d}=5^{\circ}$.

\section{EXPERIMENT STUDY}

According to Mie theory, single $180^{\circ}$ back-scattered photon by spherical scatter preserves its polarization, while multiple scattering events will depolarize the polarized light ${ }^{10}$. Assuming the Class II photon has been totally randomized at some depths, the polarization degree of the back-scattered signal will decrease to 0.5 at the cross point when Class $I$ signal 
intensity is equal to the Class II signal intensity. Therefore the change of the polarization degree gives a good indication when the multiple scattering becomes dominant.

The degree of polarization can be derived from the Stokes vector measurement. The Stokes vector $S$ of a light beam is based on six flux measurements with different analyzers in front of the detector: $S=\left[\mathrm{S}_{0}, \mathrm{~S}_{1}, \mathrm{~S}_{2}, \mathrm{~S}_{3}\right]^{\mathrm{T}}=[H+V, H-V, P-M, R-L]^{T}$, where $H, V, P, M, R$ and $L$ are the intensities of the light beam measured with a horizontal linear polarizer, a vertical linear polarizer, a $+45^{\circ}$ linear polarizer, a $135^{\circ}\left(-45^{\circ}\right)$ linear polarizer, a right circular analyzer, and a left circular analyzer in front of the detector, respectively. The superscript $T$ transposes the row vector into a column vector. Because of the following relationships: $H+V=P+M=R+L$, a Stokes vector can be determined by four independent measurements. The degree of polarization (DOP) can be calculated as:

$$
D O P=\frac{\sqrt{S_{1}^{2}+S_{2}^{2}+S_{3}^{2}}}{S_{0}},
$$

A schematic of the polarization sensitive OCT system for our studies is shown in Fig. 8. A superluminescent diode (SLD) with a center wavelength of $0.83 \mu \mathrm{m}$ and a FWHM bandwidth of $20 \mathrm{~nm}$ is used as the light source. After passing through the polarizer $\mathrm{P}$, the light is split by a nonpolarization beam splitter (NBS). The sample beam is focused into the sample by an objective lens (L1) with a N.A. of 0.15 . The reference beam passes through a variable-wave plate and is reflected back. The reflected beams from the reference and sample arms are coupled into a single-mode fiber and detected by a silicon photodiode. The flux with the reference polarization $P$ is:

$$
I_{s, P} \propto I_{o c t}^{2} / I_{r, P},
$$

where $I_{o c t}$ is the detected OCT signal intensity, $I_{r, p}$ is the intensity of the reference beam. In the experiment, the incident light is horizontal polarized. The variable-wave plate at the reference arm is adjusted to sequentially achieve the $H, V, P$ and $R$ polarization states. The light intensities of the reference arm are measured for calibration purpose. The OCT system is carefully calibrated and validated. The four reference polarization states are examined in terms of polarization purity. The

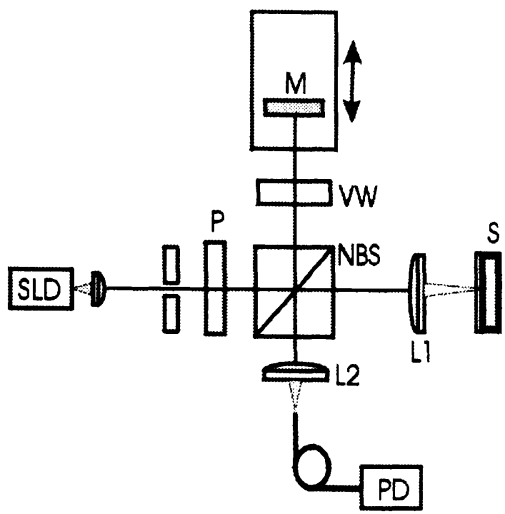

Fig.8. Schematic of the polarization-sensitive OCT system: SLD superluminescent diode; P polarizer; NBS non-polarization beam splitter; VW variable-wave plate; $M$ mirror; L1, L2 lens; PD photodiode. polarization purity is defined as $I_{\min } / I_{\max }$, where $I_{\max }$ is the signal intensity of the designed polarization state, and $I_{\min }$ is the intensity of the orthogonal polarization state. The measured $I_{\min } / I_{\max }$ is less than $0.15 \%$ for all of the polarization states.

Intralipid solution is used as the turbid media sample. A previous study ${ }^{11}$ shows that scatters in the Intralipid solution are approximately spherical. The average size is about $100 \mathrm{~nm}$ which is in the Rayleigh scattering region for the SLD wavelength of $830 \mathrm{~nm}$. In this specific experiment, the $S_{2}, S_{3}$ terms of the Stokes vector are always zero due to the symmetrical property. Therefore the DOP could be simply derived as $D O P=S_{l} / S_{0}$. The OCT signal is multiply averaged to reduce the speckle noise. Polynomial fitting is applied to each measurement to obtain a smooth curve for calculation.

A typical result is shown in Fig.9. The sample is $1 \%$ Intralipid solution. The co-polarized OCT signal initially decays exponentially. At some depth, the decay becomes slower which indicates the multiple scattering begins to cumulate. The DOP curve reveals more clearly this trend. Initially, the DOP is near 1.0 and the back-scattered light preserves its polarization. In other words, the single back-scattering signal is dominant until this point. When the depth increases more, the DOP begins to drop. If we assume that the Class I signal preserves its polarization and the Class II signal is totally depolarized beyond some depths, then there is a crossover point where DOP becomes 0.5 . At this point, the Class I signal intensity is equal to the Class II signal intensity. This crossover point can be considered as the achievable image depth for the OCT system in this specific turbid medium. The image depths $(\mathrm{DOP}=0.5)$ in the Intralipid of different concentration are measured in term of Optical Depth (OD) which is defined as $\mathrm{OD}=\mu_{\mathrm{s}} \times$ depth. The results show the achievable imaging depth is within the range of 3-4 OD's. 


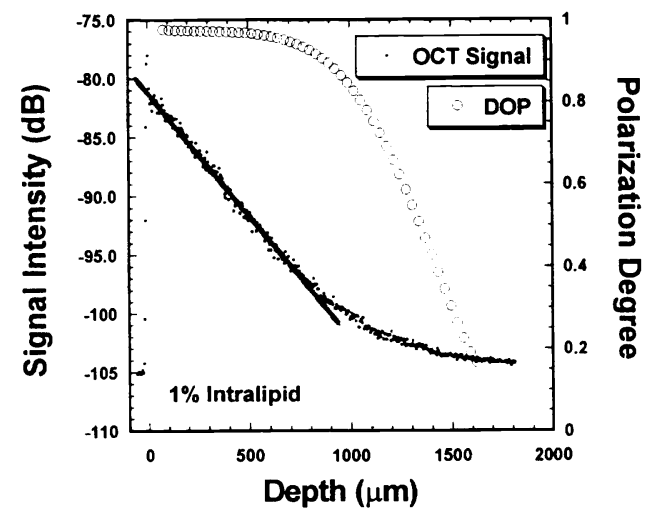

Fig.9. Co-polarized OCT signal and DOP curve from $1 \%$ Intralipid solution

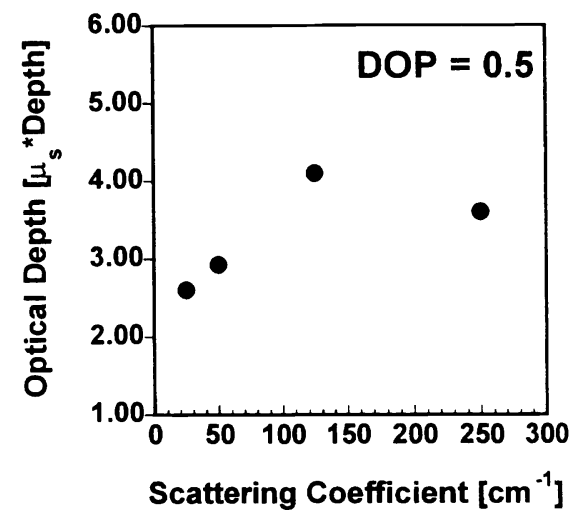

Fig.10. Imaging Depth $(\mathrm{DOP}=0.5)$ in Intralipid solution

\section{CONCLUSION}

In this paper, the angle biased Monte Carlo method has been used to study the optical coherence tomography signal in homogeneous turbid media. The OCT signal is divided into two categories: one is the light back-scattered from the target layer (Class I) and the other is the multiply scattered light from the background other than the target layer (Class II). The multiply scattered photons decrease the contrast of the OCT image and dominate the singly back-scattered component for large probing depths. The Class II signal has wider spatial and angular distributions than the Class I signal and experiences more scattering events. The average number of scattering events increases linearly with the probing depth for both the Class $I$ and Class II signals. Because of the limitation of the optical path length (Coherent Gating), the multiple scattering events associated with the Class I photons are probably forward scattering events. By using a polarization sensitive OCT, we show that the change of polarization degree can be used to indicate the dominance of the multiple-scattered signal. Experimental results show the imaging depth is limited to 3-4 OD's by the multiple scattering events. Such results may depend on the specific imaging scheme and further study is needed to achieve the optimal imaging depth.

\section{ACKNOWLEDGMENTS}

This project was sponsored in part by the National Institutes of Health grants R29 CA68562 and R01 CA71980 and by the National Science Foudation grant BES-9734491.

\section{REFERENCE}

1. D. Huang, E.A. Swanson, C.P. Lin, J.S. Schuman, W.G. Stinson, W. Chang, M.R. Hee, T. Flotte, K. Gregory, C.A. Puliafito, and J.G. Fujimoto, "Optical coherence tomography", Science 254, 1178-1181(1991).

2. D. Huang, J. Wang, C.P. Lin, C.A. Puliafito, and J.G. Fujimoto, "Micro-resolution ranging of cornea anterior chamber by optical reflectrometry," Laser Surg. Med. 11, 419-425(1991).

3. C.A. Puliafito, M.R. Hee, C.P. Lin, E.Reichel, J.S. Schuman, J.S. Duker, J.A. Izatt, E.A. Swanson, and J.G. Fujimoto, "Imaging of macular disease with optical coherence tomography", Opthalmology 120, 217-229(1995).

4. M.J. Yadlowsky, J.M. Schmitt, and R.F. Bonner, "Multiple scattering in optical coherence microscopy," Appl. Opt. 34, 5699-5707.

5. Y.T. Pan, R. Birngruber, J. Rosperich, and R. Engelhardt, "Low-coherence optical tomography in turbid tissue: theoretical analysis," Appl. Opt. 34, 6564-6574(1995). 
6. J.M. Schmitt and A. Knuttel, "Model of optical coherence tomography of heterogeneous tissue," J. Opt. Soc. Am. A. 14, 1231-1242.

7. L.H. Wang, S.L. Jacques, and L.Q. Zheng, "MCML - Monte Carlo modeling of photon transport in multi-layered tissue," Computer Methods and Programs in Biomedicine 47, 131-146(1995).

8. J.S. Hendricks and L.L. Carter, "Anisotropic angle biasing of photons," Nucl. Sci. Eng. 89, 118-130(1985).

9. J.M. Schmitt and K. Ben-Letaief, "Efficient Monte Carlo simulation of confocal microscopy in biological tissue," J. Opt. Soc. Am. A. 13, 952-961.

10. J. M. Schmitt and S. H. Xiang, Opt. Lett. 23, 1060 (1998).

11. Hugo J. van Staveren, Christian J. M. Moes, Jan van Marle, Scott A. Prahl, and Martin J. C. van Gemert, "Light scattering in Intralipid-10\% in the wavelength range of 400-1100nm", Appl. Opt. 30, 4507-4514(1991). 